\title{
The reproducing kernel thesis for lower bounds of weighted composition operators
}

\author{
I. Chalendar and J. R. Partingtonid
}

\begin{abstract}
It is shown that the property of being bounded below (having closed range) of weighted composition operators on Hardy and Bergman spaces can be tested by their action on a set of simple test functions, including reproducing kernels. The methods used in the analysis are based on the theory of reverse Carleson embeddings.
\end{abstract}

Mathematics Subject Classification. 47B33, 30H10, 32A36, $47 \mathrm{~B} 32$.

Keywords. Reproducing kernel, Weighted composition operator, Reverse Carleson measure, Hardy space, Bergman space, Test functions.

1. Introduction and notation. The reproducing kernel thesis is a term commonly used to describe a body of results that assert that the boundedness of various operators on function spaces such as the Hardy and Bergman space can be tested by their action on reproducing kernels: this is known to apply to Hankel operators, Toeplitz operators, Carleson embeddings, and - our main concern here - weighted composition operators, although not to the adjoints of weighted composition operators (see [13], also [4,11]).

Surprisingly, the reproducing kernels may also be used in some circumstances as test functions for boundedness below of certain operators, and this is the theme of this paper. We give necessary and sufficient conditions for a bounded weighted composition operator $W$ on a Hardy space $H^{p}$ or a Bergman space $A^{p}$ to be bounded below, that is, for there to exist a constant $\delta>0$ such that $\|W f\| \geq \delta\|f\|$ for all $f$ in the space. Assuming that the operator is injective, which it is except in trivial cases, this property is equivalent to the property that $W$ has closed range. Some questions remain open in the case of Bergman spaces, particularly for the case $p=2$ if the weight has infinitely many zeros. 
The results of this paper generalize in various directions earlier work from $[2,10,12,16,18]$, as will be seen below.

We write $m$ for the normalized Lebesgue measure on $\mathbb{T}$ and $A$ for the normalized area measure in $\mathbb{D}$.

2. Hardy spaces. For $h \in H^{p}=H^{p}(\mathbb{D})$ and $\psi: \mathbb{D} \rightarrow \mathbb{D}$ holomorphic we define the weighted composition operator $W_{h, \psi}$ on $H^{p}$ by

$$
W_{h, \psi} f(z)=h(z) f(\psi(z)), \quad(z \in \mathbb{D}) .
$$

If $h \in H^{\infty}$, then $W_{h, \psi}$ is automatically bounded, by Littlewood's subordination theorem, but this is not a necessary condition for boundedness.

For $\lambda \in \mathbb{D}$, we write $k_{\lambda}$ for the reproducing kernel function $z \mapsto 1 /(1-\bar{\lambda} z)$ for $z \in \mathbb{D}$. Using the (isomorphic) duality between $H^{p}$ and $H^{p^{\prime}}$, where $p^{\prime}=$ $p /(p-1)$ is the conjugate index to $p[19, \mathrm{~A} 5.7 .8]$, we see that there are constants $A, B>0$ independent of $\lambda$ such that for $1<p<\infty$ we have

$$
A\left\|\delta_{\lambda}\right\|_{\left(H^{p^{\prime}}\right)^{*}} \leq\left\|k_{\lambda}\right\|_{p} \leq B\left\|\delta_{\lambda}\right\|_{\left(H^{p^{\prime}}\right)^{*}},
$$

where $\delta_{\lambda}$ is the functional $f \mapsto f(\lambda)$. Now, the standard inner-outer factorization shows that every $f \in H^{p^{\prime}}$ can be written as $f=\theta u^{2 / p^{\prime}}$ for $\theta$ inner and $u \in H^{2}$ outer, and conversely every $g \in H^{2}$ can be written as $g=\phi v^{p^{\prime} / 2}$ for $\phi$ inner and $v \in H^{p^{\prime}}$ outer. We conclude easily that

$$
\left\|\delta_{\lambda}\right\|_{\left(H^{p^{\prime}}\right)^{*}}=\left\|\delta_{\lambda}\right\|_{H^{2}}^{2 / p^{\prime}}=\left(1-|\lambda|^{2}\right)^{-1 / p^{\prime}} .
$$

We write $\tilde{k}_{\lambda}=k_{\lambda} /\left\|k_{\lambda}\right\|_{p}$, noting that this definition depends on $p$.

As in [3] we define the measure $\mu_{h, \psi}$ on Borel subsets of $\overline{\mathbb{D}}$ by

$$
\mu_{h, \psi}(E)=\int_{\psi^{-1}(E) \cap \mathbb{T}}|h|^{p} d m,
$$

where $m$ is normalized Lebesgue measure. In [3] it is shown that $W_{h, \psi}$ is bounded on $H^{p}$ if and only if $\mu_{h, \psi}$ is a Carleson measure: this follows from the fact that

$$
\left\|W_{h, \psi} f\right\|^{p}=\int_{\overline{\mathbb{D}}}|f|^{p} d \mu_{h, \psi}
$$

for $f \in H^{p}$.

Cima, Thomson, and Wogen [2] showed that the composition operator $C_{\phi}$ on $H^{2}$ (the case $h=1, p=2$ ) has closed range if and only if the RadonNikodym derivative

$$
g_{h, \psi}=\frac{d \mu_{h, \psi}}{d m}
$$

is essentially bounded away from 0 on $\mathbb{T}$. See also [20] for another characterization. This was extended to $H^{p}$ by Galanopoulos and Panteris [10].

In [16], the result was extended to weighted composition operators on $H^{2}$. In fact a similar argument gives the full result for $H^{p}$. 
Lemma 2.1. Let $1<p<\infty$ and let $h \in H^{p}$ and $\psi: \mathbb{D} \rightarrow \mathbb{D}$ holomorphic such that $W_{h, \psi}$ is bounded. Then $W_{h, \psi}$ is bounded below if and only if $g_{h, \psi}$, as defined in (2), is essentially bounded away from 0 on $\mathbb{T}$.

Proof. It follows from (1) that

$$
\left\|W_{h, \psi} f\right\|^{p} \geq \int_{\mathbb{T}}|f|^{p} g_{h, \psi} d m,
$$

and so $W_{h, \psi}$ is bounded below if $g_{h, \psi}$ is essentially bounded away from 0 .

Conversely, if $g_{h, \psi}$ is not essentially bounded away from 0 , then for each $\epsilon>0$ there is a set $E \subset \mathbb{T}$ such that $m(E)>0$ and

$$
\int_{\psi^{-1}(E) \cap \mathbb{T}}|h|^{p} d m<\epsilon m(E) .
$$

As in [6, p. 24], for example, there exists a function $f \in H^{p}$ such that

$$
\left|f\left(e^{i \theta}\right)\right|= \begin{cases}1 & \text { if } e^{i \theta} \in E \\ \frac{1}{2} & \text { if } e^{i \theta} \in \mathbb{T} \backslash E .\end{cases}
$$

Now for $n=1,2, \ldots$ we have $\left\|f^{n}\right\|_{p} \geq m(E)^{1 / p}$ but $f^{n} \rightarrow 0$ pointwise on $\mathbb{T} \backslash E$, so

$$
\limsup _{n \rightarrow \infty}\left\|W_{h, \psi} f^{n}\right\|_{p}^{p}=\int_{\psi^{-1}(E) \cap \mathbb{T}}\left|h\left(e^{i \theta}\right)\right|^{p} d m \leq \epsilon m(E) .
$$

This leads to a reproducing kernel thesis for boundedness below on $H^{p}$.

Theorem 2.2. Let $1<p<\infty$ and let $h \in H^{p}$ and $\psi: \mathbb{D} \rightarrow \mathbb{D}$ be holomorphic with $W_{h, \psi}$ bounded. The following assertions are equivalent:

(i) $W_{h, \psi}$ is bounded below;

(ii) There exists $C>0$ such that $\left\|W_{h, \psi} k_{\lambda}\right\|_{p} \geq C\left\|k_{\lambda}\right\|_{p}$ for all $\lambda \in \mathbb{D}$.

Proof. By [14, Theorem 2.1] (see also [9]), the function $g_{h, \psi}$ is essentially bounded away from 0 if and only if there is a constant $C>0$ such that

$$
\int_{\overline{\mathbb{D}}}\left|\tilde{k}_{\lambda}(z)\right|^{p} d \mu_{h, \psi} \geq C \quad(\lambda \in \mathbb{D}) .
$$

Using (1) and Lemma 2.1 we have the result.

For unweighted composition operators, and $p=2$, this result may be found in the thesis of Luery [18].

Now for $1 \leq p<\infty$, let $\tilde{\ell}_{w} \in H^{p}$ be defined by

$$
\tilde{\ell}_{w}(z)=\frac{\left(1-|w|^{2}\right)^{1 / p}}{(1-\bar{w} z)^{2 / p}},
$$

so that $\left\|\tilde{\ell}_{w}\right\|_{H^{p}}=1$ for all $w \in \mathbb{D}$. We may use these test functions for boundedness below of weighted composition operators on $H^{p}$. 
Theorem 2.3. Let $1 \leq p<\infty, h \in H^{p}$, and $\psi: \mathbb{D} \rightarrow \mathbb{D}$ be holomorphic such that the weighted composition operator $W_{h, \psi}$ is bounded. Then $W_{h, \psi}$ is bounded below if and only if there is a constant $C>0$ such that $\left\|W_{h, \psi} \tilde{\ell}_{w}\right\| \geq C$ for all $w \in \mathbb{D}$.

Proof. Without loss of generality, we may assume that $h$ is outer, and hence non-vanishing, since if $h=\theta u$ with $\theta$ inner and $u$ outer, then the operator $W_{u, \psi}$ is bounded below if and only if $W_{h, \psi}$ is; a similar observation applies to boundedness below on test functions. The condition on test functions may be written as

$$
\int_{\mathbb{T}}|h(z)|^{p}\left|\tilde{\ell}_{w}(\psi(z))\right|^{p} d m(z) \geq C^{p}
$$

and since $h$ is non-vanishing, we may write $h(z)^{p}=\tilde{h}(z)^{2}$, where $\tilde{h} \in H^{2}$. Thus with $\tilde{k}_{w}=k_{w} /\left\|k_{w}\right\|_{2}$ we have

$$
\int_{\mathbb{T}}|\tilde{h}(z)|^{2}\left|\tilde{k}_{w}(\psi(z))\right|^{2} d m(z) \geq C^{p}
$$

for all $w \in \mathbb{D}$, and so the weighted composition operator $W_{\tilde{h}, \psi}$ is bounded below on $H^{2}$, by Theorem 2.2. It now follows from Lemma 2.1 that $W_{h, \psi}$ is bounded below on $H^{p}$.

As a corollary of Theorem 2.2 we have a reproducing kernel thesis for boundedness below of composition operators $C_{\Phi}$ on the right half-plane $\mathbb{C}_{+}$. Note that these operators are not automatically bounded, but an exact expression for their norm is given in [7].

By means of a unitary equivalence between $H^{2}(\mathbb{D})$ and $H^{2}\left(\mathbb{C}_{+}\right)$induced by the self-inverse Möbius bijection $M(z)=(1-z) /(1+z)$, namely,

$$
(V f)(s)=\frac{1}{\sqrt{\pi}(1+s)} f\left(\frac{1-s}{1+s}\right), \quad f \in H^{2}(\mathbb{D}), \quad s \in \mathbb{C}_{+},
$$

the composition operator $C_{\Phi}$ on $H^{2}\left(\mathbb{C}_{+}\right)$is seen to be unitarily equivalent to the weighted composition operator $W_{h, \phi}$ on $H^{2}(\mathbb{D})$, where $h(z)=\frac{1+\phi(z)}{1+z}$ and $\phi=M \circ \Phi \circ M($ see $[1,16])$.

Let $\tilde{K}_{w}$, given by

$$
\tilde{K}_{w}(s)=\frac{(2 \pi \operatorname{Re} w)^{1 / 2}}{s+\bar{w}},
$$

denote the normalized reproducing kernel at $w \in \mathbb{C}_{+}$. Since $\left\langle F, V k_{\lambda}\right\rangle=$ $\left(V^{-1} F\right)(\lambda)$ for $F \in H^{2}\left(\mathbb{C}_{+}\right)$, we conclude that $V \tilde{k}_{\lambda}=\tilde{K}_{w}$, where $w=M(\lambda)$, and hence obtain the following corollary, which can also be proved fairly directly.

Corollary 2.4. If the composition operator $C_{\Phi}$ is bounded on $H^{2}\left(\mathbb{C}_{+}\right)$, then it is bounded below if and only if there is a constant $C>0$ such that $\left\|C_{\Phi} \widetilde{K}_{w}\right\| \geq C$ for all $w \in \mathbb{C}_{+}$. 
Clearly similar results hold for weighted composition operators, and also for $H^{p}\left(\mathbb{C}_{+}\right)$for other values of $p$.

Another easy corollary of the main theorem of [14] is a reproducing kernel thesis for Toeplitz operators. If $J: H^{2} \rightarrow L^{2}(\mathbb{T}, \mu)$ is a bounded (Carleson) embedding, then it is clearly bounded below if and only if $J^{*} J$ is bounded below as an operator on $H^{2}$ (consider $\left.\left\langle J^{*} J x, x\right\rangle\right)$.

Corollary 2.5. Suppose that $h \in L^{\infty}(\mathbb{T})$ with $h \geq 0$ a.e. Then the Toeplitz operator $T_{h}: f \mapsto P_{H^{2}}(h . f)$ is bounded below if and only if it is bounded below on normalized reproducing kernels.

Proof. Let $\mu$ denote the measure with Radon-Nikodym derivative $h$, so that

$$
\left\langle J^{*} J f, g\right\rangle=\int_{\mathbb{T}} f \bar{g} h d m=\left\langle T_{h} f, g\right\rangle
$$

for $f, g \in H^{2}$. Since we can test $J^{*} J$ on normalized reproducing kernels, by [18], or indeed Theorem 2.2, the result follows.

3. Bergman spaces. We now consider weighted composition operators $W_{h, \psi}$ acting on the Bergman space $A^{p}=A^{p}(\mathbb{D})$. Once again a measure is associated with such an operator, this time $\mu_{h, \psi}^{p}$ defined on Borel subsets of the disc by

$$
\mu_{h, \psi}^{p}(E)=\int_{\psi^{-1}(E)}|h(z)|^{p} d A(z)
$$

and we have

$$
\left\|W_{h, \psi} f\right\|^{p}=\int_{\mathbb{D}}|f(z)|^{p} d \mu_{h, \psi}^{p}(z) .
$$

This is done in $[16$, Lem. 3.1] for the case $p=2$, but the argument works for all $p$. It follows that $W_{h, \psi}$ is bounded and bounded below if and only if $\mu_{h, \psi}^{p}$ satisfies the Carleson and reverse Carleson properties. The unweighted case of this result for $p=2$ may be found in [20].

We begin with the case $p=2$ and write $\mu_{h, \psi}$ for $\mu_{h, \psi}^{2}$ and $g_{h, \psi}$ for the Radon-Nikodym derivative of $\mu_{h, \psi}$. We have, using [17, Corollary 1], that $W_{h, \psi}$ is bounded below if and only if there exist constants $\delta, C>0$ such that

$$
A\left(D \cap\left\{z \in \mathbb{D}: g_{h, \psi}(z)>\delta\right\}\right) \geq C A(D \cap \mathbb{D})
$$

for all discs $D$ with centres on $\mathbb{T}$. (See [16, Thm. 3.1].)

More recently, Ghatage and Tjani [12] have analysed the unweighted case by means of the Berezin transform: in our context we define it by

$$
\tilde{\mu}(h, \psi)(w)=\int_{\mathbb{D}}\left|\tilde{k}_{w}(z)\right|^{2} d \mu_{h, \psi}(w)=\left\|W_{h, \psi} \tilde{k}_{w}\right\|^{2},
$$

where now $\tilde{k}_{w}$ is the normalized Bergman kernel,

$$
\tilde{k}_{w}(z)=\frac{1-|w|^{2}}{(1-\bar{w} z)^{2}}, \quad w, z \in \mathbb{D} .
$$


The following theorem gives an extension of [12] to weighted composition operators.

Theorem 3.1. For a bounded weighted composition operator $W_{h, \psi}$ on $A^{2}(\mathbb{D})$ the following conditions are equivalent:

(i) $W_{h, \psi}$ is bounded below;

(ii) $\mu_{h, \psi}$ satisfies the reverse Carleson condition;

(iii) $\tilde{\mu}(h, \psi)(w)$ is bounded away from zero; that is, there is a constant $C>0$ such that $\left\|W_{h, \psi} \tilde{k}_{w}\right\| \geq C$ for all $w$.

Proof. The equivalence of (i) and (ii) is given in [16, Lem. 3.1]; the equivalence of (ii) and (iii) is contained in [12, Theorem 4.1], which asserts that a measure satisfies the reverse Carleson condition if and only if its Berezin transform is bounded away from 0, together with (5).

Remark 3.2. In the case of weighted composition operators on weighted Bergman spaces $A_{\alpha}^{2}$, with $\alpha>-1$ and the norm given by

$$
\|f\|_{A_{\alpha}^{2}}^{2}=\int_{\mathbb{D}}|f(z)|^{2}\left(1-|z|^{2}\right)^{\alpha} d A(z),
$$

we still have the equivalence of (i) and (ii) in Theorem 3.1, since the proof of Lemma 3.1 in [16] is easily seen to extend to this situation. However, at present we do not know whether the equivalence with (iii) still holds.

As with Corollary 2.4 we may obtain a corollary for composition operators on the Bergman space of $\mathbb{C}_{+}$. We note that the norm of a bounded composition operator on $A^{2}\left(\mathbb{C}_{+}\right)$is given in [8]. In the following result $\widetilde{K}_{w}$ is the normalized reproducing kernel for $A^{2}\left(\mathbb{C}_{+}\right)$.

Corollary 3.3. If the composition operator $C_{\Phi}$ is bounded on $A^{2}\left(\mathbb{C}_{+}\right)$then it is bounded below if and only if there is a constant $C>0$ such that $\left\|C_{\Phi} \widetilde{K}_{w}\right\| \geq C$ for all $w \in \mathbb{C}_{+}$.

Since the proof is very similar to the proof of Corollary 2.4, we omit it.

Now for $1 \leq p<\infty$, let $\tilde{\ell}_{w} \in A^{p}$ be defined by

$$
\tilde{\ell}_{w}(z)=\frac{\left(1-|w|^{2}\right)^{2 / p}}{(1-\bar{w} z)^{4 / p}},
$$

so that $\left\|\tilde{\ell}_{w}\right\|_{A^{p}}=1$ for all $w \in \mathbb{D}$. We may use these test functions for boundedness below of weighted composition operators on $A^{p}$. The following theorem corresponds to Theorem 2.3 for the Hardy space, but requires a supplementary condition on $h$, as we do not have a suitable inner-outer factorization available.

Theorem 3.4. Let $1 \leq p<\infty, h \in A^{p}$ with at most finitely-many zeros, and $\psi: \mathbb{D} \rightarrow \mathbb{D}$ holomorphic such that the weighted composition operator $W_{h, \psi}$ is bounded. Then $W_{h, \psi}$ is bounded below if and only if there is a constant $C>0$ such that $\left\|W_{h, \psi} \tilde{\ell}_{w}\right\| \geq C$ for all $w \in \mathbb{D}$. 
Proof. As in the proof of Theorem 2.3, we may assume without loss of generality that $h$ has no zeros. This time we divide out its zeros by a contractive divisor $G$, as in $[5,15]$. Since $G$ is analytic on a neighbourhood of the disk, it is also bounded, and thus plays the same role as the inner function $\theta$ did in the Hardy space. That is, $W_{h, \psi}$ and $W_{h / G, \psi}$ are both bounded below (or not) together.

The condition on test functions may be written as

$$
\int_{\mathbb{D}}|h(z)|^{p}\left|\tilde{\ell}_{w}(\psi(z))\right|^{p} d A(z) \geq C^{p},
$$

and since $h$ is non-vanishing we may write $h(z)^{p}=\tilde{h}(z)^{2}$, where $\tilde{h} \in A^{2}$. Thus

$$
\int_{\mathbb{D}}|\tilde{h}(z)|^{2}\left|\tilde{k}_{w}(\psi(z))\right|^{2} d A(z) \geq C^{p}
$$

for all $w \in \mathbb{D}$, and so the weighted composition operator $W_{\tilde{h}, \psi}$ is bounded below on $A^{2}$, by Theorem 3.1 .

Looking at (3) and (4), noting that $\mu_{h, \psi}^{p}=\mu_{\tilde{h}, \psi}^{2}$, and observing that Luecking's condition for a reverse Carleson measure [17, Cor. 1] is independent of $p$, we see that $W_{h, \psi}$ is bounded below on $A^{p}$.

It would be interesting to know whether Theorem 3.4 extends to the case when $h$ has infinitely-many zeros, and the corresponding contractive divisor $G$ may not be bounded.

Open Access. This article is distributed under the terms of the Creative Commons Attribution 4.0 International License (http://creativecommons.org/licenses/ by/4.0/), which permits unrestricted use, distribution, and reproduction in any medium, provided you give appropriate credit to the original author(s) and the source, provide a link to the Creative Commons license, and indicate if changes were made.

Publisher's Note Springer Nature remains neutral with regard to jurisdictional claims in published maps and institutional affiliations.

\section{References}

[1] Chalendar, I., Partington, J.R.: On the structure of invariant subspaces for isometric composition operators on $H^{2}(\mathbb{D})$ and $H^{2}\left(\mathbb{C}_{+}\right)$. Arch. Math. (Basel) 81(2), 193-207 (2003)

[2] Cima, J.A., Thomson, J., Wogen, W.: On some properties of composition operators. Indiana Univ. Math. J. 24, 215-220 (1974/75)

[3] Contreras, M.D., Hernández-Díaz, A.G.: Weighted composition operators on Hardy spaces. J. Math. Anal. Appl. 263(1), 224-233 (2001)

[4] Cučković, Z., Zhao, R.: Weighted composition operators between different weighted Bergman spaces and different Hardy spaces. Ill. J. Math. 51(2), 479$498(2007)$ 
[5] Duren, P., Khavinson, D., Shapiro, H.S., Sundberg, C.: Contractive zero-divisors in Bergman spaces. Pacific J. Math. 157(1), 37-56 (1993)

[6] Duren, P.L.: Theory of $H^{p}$ Spaces, 2nd edn. Dover Publications, Mineola (2003)

[7] Elliott, S., Jury, M.T.: Composition operators on Hardy spaces of a half-plane. Bull. Lond. Math. Soc. 44(3), 489-495 (2012)

[8] Elliott, S.J., Wynn, A.: Composition operators on weighted Bergman spaces of a half-plane. Proc. Edinb. Math. Soc. (2) 54(2), 373-379 (2011)

[9] Fricain, E., Hartmann, A., Ross, W.T.: A survey on reverse Carleson measures. In Harmonic analysis, function theory, operator theory, and their applications, Theta Ser. Adv. Math, Theta, Bucharest, pp. 91-123 (2017)

[10] Galanopoulos, P., Panteris, K.: Closed range composition operators on Hardy spaces (2017). Preprint, arxiv:1711.05047

[11] Gallardo-Gutiérrez, E.A., Kumar, R., Partington, J.R.: Boundedness, compactness and Schatten-class membership of weighted composition operators. Integral Equ. Oper. Theory 67(4), 467-479 (2010)

[12] Ghatage, P., Tjani, M.: Closed range composition operators on Hilbert function spaces. J. Math. Anal. Appl. 431(2), 841-866 (2015)

[13] Harper, Z.: Applications of the discrete Weiss conjecture in operator theory. Integral Equ. Oper. Theory 54(1), 69-88 (2006)

[14] Hartmann, A., Massaneda, X., Nicolau, A., Ortega-Cerdà, J.: Reverse Carleson measures in Hardy spaces. Collect. Math. 65(3), 357-365 (2014)

[15] Hedenmalm, H.: A factorization theorem for square area-integrable analytic functions. J. Reine Angew. Math. 422, 45-68 (1991)

[16] Kumar, R., Partington, J.R.: Weighted composition operators on Hardy and Bergman spaces. In: Recent Advances in Operator Theory, Operator Algebras, their Applications, pp. 157-167, Oper. Theory Adv. Appl., vol. 153, Birkhäuser, Basel (2005)

[17] Luecking, D.H.: Inequalities on Bergman spaces. Ill. J. Math. 25(1), 1-11 (1981)

[18] Luery, K.: Composition operators on Hardy spaces of the disk and half-plane. Ph.D. thesis, University of Florida, Gainesville (2013)

[19] Nikolski, N.K.: Operators, Functions, and Systems: An Easy Reading, vol. 1. Hardy, Hankel, and Toeplitz. Translated from the French by Andreas Hartmann. Mathematical Surveys and Monographs, 92, American Mathematical Society, Providence, RI (2002)

[20] Zorboska, N.: Composition operators with closed range. Trans. Am. Math. Soc. 344(2), 791-801 (1994)

\section{Chalendar}

Université Paris Est, LAMA, (UMR 8050),

UPEM, UPEC, CNRS

77454 Marne-la-Vallée

France

e-mail: isabelle.chalendar@u-pem.fr 
J. R. PARtington

School of Mathematics

University of Leeds

Leeds LS2 9JT

UK

e-mail: J.R.Partington@leeds.ac.uk

Received: 18 December 2018 